\title{
Molecular dysfunctions in acute myeloid leukemia revealed by integrated analysis of microRNA and transcription factor
}

\author{
XIAO-CONG LIN ${ }^{1}$, YONG XU², GUO-PING SUN ${ }^{2}$, JIN-LI WEN ${ }^{3}, \mathrm{NING} \mathrm{LI}^{4}$, \\ YU-MING ZHANG ${ }^{4}$, ZHI-GANG YANG ${ }^{4}$, HAI-TAO ZHANG ${ }^{1}$ and YONG DAI $^{3}$ \\ ${ }^{1}$ Institute of Biochemistry and Molecular Biology, Guangdong Medical University, Zhanjiang, Guangdong 524023; \\ ${ }^{2}$ Shenzhen Pingshan People's Hospital, Shenzhen, Guangdong 518118; ${ }^{3}$ Clinical Medical Research Center, \\ Shenzhen People's Hospital, Shenzhen, Guangdong 518020; ${ }^{4}$ Department of Hematology, Affiliated \\ Hospital of Guangdong Medical University, Zhanjiang, Guangdong 524001, P.R. China
}

Received December 13, 2015; Accepted January 19, 2016

DOI: $10.3892 /$ ijo.2016.3489

\begin{abstract}
Acute myeloid leukemia (AML) is a heterogenic hematological malignancy with pathogenesis that has yet to be elucidated. MicroRNAs (miRNAs) and transcription factors (TFs) are two major regulators of gene expression, which may play important roles in the etiology of AML. However, the global regulation of gene expression in AML, involving miRNAs and TFs, still remains elusive. To characterize the global role of miRNAs and TFs in AML pathogenesis, large scale expression profiling of miRNA and TF was performed using miRNA sequencing and TF array technology, respectively, and validated by qPCR. In the present study, 308 miRNAs and 84 TFs were identified to be differentially expressed (fold-change $\geq 2.0$ ) in AML samples relative to their controls. After integrating the expression profiling data into bioinformatic analysis, we identified 1,462 miRNA-gene pairs, 982 TF-gene pairs and 296 TF-miRNA pairs. By merging these regulatory relations together, we constructed a comprehensive AML-specific miRNA-TF regulatory network. In this network, we identified 22 hub miRNAs and 11 hub TFs. KEGG
\end{abstract}

Correspondence to: Professor Yong Dai, Clinical Medical Research Center, Shenzhen People's Hospital, 1017 Dongmen North Road, Shenzhen, Guangdong 518020, P.R. China

E-mail: daiyong22@aliyun.com

Abbreviations: AML, acute myeloid leukemia; miRNA, microRNA; TF, transcription factor; pri-miRNA, primary miRNA; pre-miRNA, precursor miRNA; 3'-UTR 3'-untranslated region; TFBS, transcription factor binding site; TSS, transcription start sites; GO, Gene Oncology; KEGG pathway, Kyoto Encydopedia of Gene and Genomes; qPCR, quantitative PCR; PAGE, polyacrylamide gel electrophoresis; HRP, streptavidin-conjugated horseradish peroxidase; lncRNA, long non-coding RNA; FBL, feedback loop; FFL, feed-forward loop; DAVID, the database for annotation, visualization and integrated discovery

Key words: acute myeloid leukemia, microRNA, transcription factor, sequencing, microarray pathway analysis showed that the network nodes were significantly enriched in 33 different pathways, of which the AML pathway was the most significant. After analyzing the topology of the subnetwork, we propose that TCF3 was a potential key regulator in this regulatory network. In conclusion, this is the first study perform on global expression profiling of miRNAs and TFs relating to AML. These results may enhance our understanding of the molecular mechanisms underlying AML and provide potential targets for future therapeutics.

\section{Introduction}

Acute myeloid leukemia (AML) is an aggressive hematological malignancy accounting for $\sim 80 \%$ of all adult leukemia cases, and encompasses $15-20 \%$ of leukemia cases in children $(1,2)$. It is estimated that nearly $15 \%$ of mortality among patients with hematologic malignancy were attributed to AML (3), and more than 13,000 new cases were diagnosed each year in the USA (4), thus, making AML a major cause of hematologic disorders. AML is usually characterized by malignant clonal proliferation of hematopoietic stem cells and progenitors (blasts) in the bone marrow with the complete or partial blockage at different stages of myeloid differentiation $(1,2)$. Despite advances in chemotherapy and hematopoietic stem cell transplantation, the outcome of AML patients have not improved substantially in the last four decades, with an overall 5-year survival rate of $\sim 25 \%$ (1,2,5). Relapse occurs frequently, as $\sim 50-70 \%$ of AML patients who achieve complete remission within three years after frontline therapy (2). Among these relapsed patients, only one-third are salvageable with current treatment regimens (5). Therefore, it is urgently needed to search for new therapeutic targets for AML. Securing further advances in therapy is dependent on our increasing understanding of the factors and molecular mechanisms of leukemogenesis in AML. Currently, insight into the pathogenesis of AML has largely come from the investigations of cytogenetic abnormalities and molecular genetic mutations. Many chromosomal structural aberrations and somatic mutations have been identified, which include rearrangements of RUNX1-RNNX1T1, CBFB-MYH11, PML-RARA and MLL gene (6), mutations of CEBPA, NPM1, FLT3, DNMT3A, TET2, IDH1/2 and ASXL1 gene $(1,7,8)$. 
Despite the pathological role and prognostic implications of these genes have been well elucidated, understanding of the multistep pathogenesis of AML remains limited. Specifically, the regulatory networks of AML gene expression are still unclear.

miRNAs are small non-protein coding RNA molecules of $\sim 19-25$ nucleotides that mediate post-transcriptional regulation of gene expression level (9). Pri-miRNAs are transcribed by RNA polymerase II and subsequently processed by the Drosha-DGCR8 enzyme complex in the nucleus to form $\sim 70 \mathrm{nt}$ hairpin pre-miRNAs. Finally, pre-miRNAs were cleaved in the cytoplasm by the endoribonuclease Dicer to yield mature miRNAs. The mature miRNA may bind to the 3'-UTR of target mRNAs through its seed region, which leads to mRNA degradation or inhibition of translation (10). miRNAs play important roles in a variety of biological processes and are implicated in the initiation and progression of many types of human cancers, including AML $(11,12)$. It has been reported that ectopic expression of miR-29a would induce hematopoietic stem cells/progenitors progresses to AML in a mouse model (13). High expression of miR-100 and miR-375 was found in pediatric AML patients, which were correlated with poorer relapse-free and overall survival $(14,15)$. miR-193a expression is downregulated in AML1/ETO-positive leukemia cells, suppression of miR193a expands the oncogenic activity of the fusion protein AML1-ETO involving a feedback circuitry in miR-193a and AML1-ETO/DNMTs/HDACs (16). Recently, increased miR181a expression was shown to be associated with improved prognosis in cytogenetically normal AML. In xenograft mouse models of AML, ectopic miR-181a expression inhibits tumor growth (17). Although the aberrant expression and molecular function of AML-related miRNAs were reported in these investigations, the global miRNA regulatory network in AML remains unknown.

TFs are trans-acting protein factors that control the transcription of target genes through specifically binding to the TFBS located within promoter region of target genes (18). As two major types of regulators of gene expression, TFs and miRNAs are able to tightly coordinate to ensure precise and accurate gene expression. TF and miRNA may reciprocally regulate one another and both can regulate the expression of target genes in a combinatorial manner $(18,19)$. It has been reported that miR-223 and transcription factor E2F1 regulate each other to form negative feedback loop in AML (20). Furthermore, transcription factor $\mathrm{C} / \mathrm{EBP} \alpha$ can exert its effects by inducing miR-30c inactivating the Notch1 protein and enhancing granulocytic differentiation in AML (21). Nevertheless, global regulation of gene expression involved in the TFs and miRNAs in AML are still poorly understood. Therefore, integrated analysis correlating changes in the expression patterns of miRNAs and TFs, as it relates to AML pathogenesis, requires examination.

In the present study, we analyzed miRNA and TF expression profiles in bone marrow samples of AML patients and control groups, utilizing miRNA sequencing and TF array technology, respectively. Based on a combined strategy of the target prediction of miRNAs and TFs, we integrated differentially expressed miRNAs, AML candidate genes and differentially expressed TFs to construct a comprehensive
Table I. Clinical characteristics of AML patients and controls.

\begin{tabular}{lcc}
\hline Characteristics & $\begin{array}{c}\text { AML patients } \\
(\mathrm{n}=15)\end{array}$ & $\begin{array}{c}\text { Control patients } \\
(\mathrm{n}=10)\end{array}$ \\
\hline $\begin{array}{l}\text { Age (years) } \\
\text { Gender (n) }\end{array}$ & $18-77$ (mean 40) & $10-73$ (mean 39) \\
Male & 9 & 6 \\
Female & 6 & 4 \\
FAB subtypes (n) & & \\
M0 & 1 & - \\
M1 & 3 & - \\
M2 & 5 & - \\
M3 & 2 & - \\
M4 & 2 & - \\
M5 & 1 & - \\
M6 & 1 & - \\
Leukemic blasts & $30-99($ mean 78$)$ & \\
in bone marrow (\%) & & \\
Disease (n) & & \\
AML & - & \\
Unexplained anemia & - & \\
Unexplained fever & - & \\
\hline
\end{tabular}

miRNA-TF mediated regulatory network specifically for AML. GO and pathway analysis was performed to determine associated functions and signaling pathway of the network nodes. After calculating the number of the node degree, we found some hub miRNAs and TFs, and further investigated their regulation in the subnetwork. Our results demonstrate that the altered expression levels of miRNAs and TFs may have implications in AML pathogenesis, and that integrative analysis of miRNAs and TFs may provide a new foundation for molecularly targeted therapy of AML in the future.

\section{Materials and methods}

Patient samples and ethics statement. A total of 25 bone marrow biopsy samples, including 15 patients with newly diagnosed and untreated AML and 10 age-matched controls, were enrolled in the study. The AML samples were collected at Shenzhen People's Hospital (Shenzhen, China) before any chemo- or radiotherapeutic treatment. Diagnosis and classification of AML were determined according to the French-American-British classification system $(22,23)$. To serve as controls, the samples with normal bone marrow morphology were provided from 10 patients with unexplained anemia or fever by the Affiliated Hospital of Guangdong Medical University (Zhanjiang, China). All the clinical characteristics of AML patients and controls are shown in Table I. The study was carried out according to the Helsinki convention criteria and approved by the ethics committee of the Shenzhen People's Hospital and the Affiliated Hospital of Guangdong Medical University. Written informed consent to use biological samples and clinical data was obtained from patients or their parents. 
Table II. Primers used for cDNA synthesis of miRNAs.

miRNAs

miR-9-5p

miR-155-5p

miR-100-5p

miR-223-3p

miR-16-5p

miR-106b-5p

U6 snRNA
Primer for cDNA synthesis (5'-3')
GTCGTATCCAGTGCGTGTCGTGGAGTCGGCAATTGCACTGGATACGACTCATACA GTCGTATCCAGTGCGTGTCGTGGAGTCGGCAATTGCACTGGATACGACACCCCTA GTCGTATCCAGTGCGTGTCGTGGAGTCGGCAATTGCACTGGATACGACCACAAG GTCGTATCCAGTGCGTGTCGTGGAGTCGGCAATTGCACTGGATACGACTGGGGTA GTCGTATCCAGTGCGTGTCGTGGAGTCGGCAATTGCACTGGATACGACCGCCAAT GTCGTATCCAGTGCGTGTCGTGGAGTCGGCAATTGCACTGGATACGACATCTGCA CGCTTCACGAATTTGCGTGTCAT
Total RNA extraction. Total RNA was extracted using TRIzol reagent (Invitrogen, Carlsbad, CA, USA) and a RNeasy kit (Qiagen, Hilden, Germany) following manufacturer's instructions, to include a DNase digestion step. RNA purity and concentration were determined using a NanoDrop ND-1000 spectrophotometer (NanoDrop Technologies, Wilmington, DE, USA) measuring absorbance at 230, 260 and $280 \mathrm{~nm}$.

miRNA sequencing and data analysis. Total RNA was ligated sequentially to 3' and 5' RNA adapters using T4 RNA ligase (Promega, Madison, WI, USA). Ligation products were then reverse-transcribed and PCR amplified using Illumina's proprietary RT primers and amplification primers (Illumina, San Diego, CA, USA). Subsequently, $135-155$ bp PCR amplified fragments (correspond to $\sim 15-35 \mathrm{nt}$ small RNAs) were isolated and purified from the PAGE gels. After quantified with Agilent 2100 Bioanalyzer (Agilent Technologies, Palo Alto, CA, USA), the completed libraries were diluted to a final concentration of $8 \mathrm{pM}$ and loaded on the Illumina cBot for cluster generation using TruSeq Rapid SR cluster kit (Illumina) according to the manufacturer's protocol. Finally, the sequencing was carried out on Illumina HiSeq 2000 using TruSeq Rapid SBS kit (Illumina) as recommended by the manufacturer.

After sequencing, image analysis and base calling were carried out using Off-Line Basecaller software (version V1.8.0; Illumina). The low quality reads were removed from raw sequencing reads by Solexa CHASTITY quality control filter. Subsequently, 3' adapter sequences were deleted from clean reads and the reads that were shorter than $15 \mathrm{nt}$ were excluded from further analysis. To identify miRNAs, the 3'-adapter-trimmed-reads were aligned to known human premiRNAs in the miRBase database (Release 19.0, http://www. mirbase.org/) using Novoalign software (Version v2.07.11, http://www.novocraft.com/) with at most one mismatch. In order to correct for the difference in read counts between samples, the read counts per miRNA in each sample were normalized to tpm (the clone number of transcripts per million based on the sum number of reads aligned to known human pre-miRNAs in miRBase 19.0). Differentially expressed miRNAs may be identified by a fold-change filtering. The threshold is fold-change $\geq 2.0$ (Based on the normalized most abundant tag counts).

Transcription factor/DNA array and computational analysis. The transcription factor/DNA array analysis was carried out using TranSignal ${ }^{\mathrm{TM}}$ Protein/DNA Combo Arrays (Panomics,
Redwood City, CA, USA) following the protocols provided by the manufacturer, which included 345 major transcription factors. Briefly, nuclear proteins were extracted using the Panomics nuclear extract kit (Panomics) and protein concentrations were determined by BCA protein assay kit (KangChen Bio-Tech Inc., Shanghai, China). Nuclear extract $(10 \mu \mathrm{g})$ were incubated with $10 \mu \mathrm{l}$ of biotin-labelled DNA binding oligonucleotides (TranSignal ${ }^{\mathrm{TM}}$ Probe Mix) for $30 \mathrm{~min}$ at $15^{\circ} \mathrm{C}$ to allow the formation of transcription factor/ DNA complexes. Such complexes were isolated from the free probes by spin column separation system (Panomics). The bound DNA probes were extracted, denatured and hybridized to oligonucleotides (representing 345 consensus binding sequences for TFs) on TransSignal array membrane at $42^{\circ} \mathrm{C}$ overnight. Subsequently, the blots were washed and incubated with HRP and HRP substrate working solution (Millipore, Billerica, MA, USA), and exposed to Hyperfilm ECL (Amersham Pharmacia Biotech, Uppsala, Sweden). Hybridization signals were detected using GBoX Imaging System (Syngene, Cambridge, UK), and quantitative analysis of the resulting spots were performed using the ScanAlyze software (version 1.0.3, http://graphics.stanford.edu/software/ scanalyze/). Any spot showing at least 2-fold increase or decrease is considered significant.

Quantitative PCR. Total RNA from each sample was reverse transcribed to generate cDNA with gene-specific primer (for miRNAs) and oligo dT primer (for TFs) using a Superscript ${ }^{\mathrm{TM}}$ III reverse transcriptase kit (Invitrogen, Carlsbad, CA, USA) in accordance with the manufacturer's instructions. QPCR was carried out in a total reaction volume of $10 \mu \mathrm{l}$, including $2 \mu \mathrm{l}$ of template cDNA, $5 \mu \mathrm{l}$ of $2 \mathrm{X}$ SYBR-Green PCR Master Mix (Applied Biosystems, Foster City, CA, USA), $0.5 \mu 1$ of PCR forward primer $(10 \mu \mathrm{M}), 0.5 \mu \mathrm{l}$ of PCR reverse primer $(10 \mu \mathrm{M})$ and $2 \mu \mathrm{l}$ of double-distilled water. The reactions were incubated at $95^{\circ} \mathrm{C}$ for $10 \mathrm{~min}$, followed by 40 cycles at $95^{\circ} \mathrm{C}$ for $10 \mathrm{sec}, 60^{\circ} \mathrm{C}$ for $60 \mathrm{sec}$. All reactions were run in triplicate. After PCR amplification, melt curve analysis was carried out to determine the reaction specificity. Human U6 snRNA and 18S rRNA was used to normalize the expression levels of miRNAs and TFs, respectively. Expression fold-changes were calculated using the $2^{-\Delta \Delta \mathrm{Ct}}$ method (24). The differences in gene expression levels between AML samples and controls were compared using the Student's t-test. Statistical significance was set at $\mathrm{P}<0.05$. All primers used in cDNA synthesis and qPCR are shown in Tables II, III and IV. 
Table III. Primers used for real-time quantitative PCR of miRNAs.

\begin{tabular}{lllc}
\hline miRNAs & \multicolumn{1}{c}{ Sense primer (5'-3') } & \multicolumn{1}{c}{ Antisense primer (5'-3') } & Product (bp) \\
\hline miR-9-5p & GGGGGTCTTTGGTTATCTA & CAGTGCGTGTCGTGGA & 68 \\
miR-155-5p & GGGGTAATGCTAATCGTGA & CAGTGCGTGTCGTGGAG & 66 \\
miR-100-5p & GCAACCCGTAGATCCGAA & CAGTGCGTGTCGTGGAGT & 62 \\
miR-223-3p & GGGGTGTCAGTTTGTCAAA & CAGTGCGTGTCGTGGAGT & 66 \\
miR-16-5p & GGGTAGCAGCACGTAAATA & CAGTGCGTGTCGTGGAGT & 65 \\
miR-106b-5p & GGGGGTAAAGTGCTGACAGT & GTGCGTGTCGTGGAGTCG & 64 \\
U6 snRNA & GCTTCGGCAGCACATATACTAAAAT & CGCTTCACGAATTTGCGTGTCAT & 89 \\
\hline
\end{tabular}

Table IV. Primers used for real-time quantitative PCR of TFs.

\begin{tabular}{lllc}
\hline TFs & \multicolumn{1}{c}{ Sense primer (5'-3') } & \multicolumn{1}{c}{ Antisense primer (5'-3') } & Product (bp) \\
\hline MYC & ACACATCAGCACAACTACGC & CCTCTTGACATTCTCCTCGGT & 159 \\
NFKB1 & ACTGGCTGAGCGGATGCATC & TGCTGTGGTCAGAAGGAATG & 165 \\
NR2F1 & ATCGAGAGCCTGCAGGAGAA & CTACCAAACGGACGAAGAAGAG & 163 \\
FOXO1 & GCAACTACAGCCAAAATCAC & TCAGAGAGCTACCAAGGATTC & 152 \\
FOXL1 & TGAGGTTTGATGGCAGGAAT & GATTTTCGTTGCAGACCTCTTC & 171 \\
TFAP2A & TTGGGTACGTGTGCGAAA & TCTGTTTTGTAGCCAGGAGCAT & 120 \\
18S rRNA & CCTGGATACCGCAGCTAGGA & GCGGCGCAATACGAATGCCCC & 112 \\
\hline
\end{tabular}

Association analysis of the different expression of miRNAs and TFs. Target gene prediction for different expression miRNAs. Differentially expressed miRNAs (fold change, $\geq 2.0$ ) were subjected to bioinformatic analysis for target gene prediction mainly by combinatorial utilization of four different online databases, including miRanda (version v5, http://www.ebi. ac.uk/enright-srv/microcosm/htdocs/targets/v5/), TargetScan (Release 6.2, http://www.targetscan.org/), PicTar (Release 2007, http://pictar.mdc-berlin.de/cgi-bin/PicTar_vertebrate. cgi) and miRTarBase (Release 4.5, http://mirtarbase.mbc.nctu. edu.tw/) $(25,26)$. To decrease the number of false-positive results, we integrated the predicted targets from miRanda, TargetScan and PicTar based on sequence complementarity, evolutionary conservation and free energy of RNA duplexes, which predicted by at least two of three databases were accepted as positive (25). (TFs were treated as genes when predicting miRNA $\rightarrow$ TF regulatory relations). Subsequently, we merged the results with experimentally validated miRNA targets from miRTarBase.

Combined AML candidate genes with miRNA target prediction. To avoid the redundancy, AML-associated genes were obtained from the MalaCards database (version 1.08.564, http://www.malacards.org/, MalaCards ID: LKM061) (27) and overlapped with the predicted miRNA targets above to form a miRNA $\rightarrow$ gene regulatory relations. The extracted miRNA and target gene pairs were then subjected to transcription factor binding site (TFBS) analysis.

TFBS prediction for different expression miRNAs and its pair target genes. To retrieve predicted TFBS information, We utilized the data underlying the TFBS Conserved Track (http://
genome.ucsc.edu/cgi-bin/hgTables?hgsid=350051003\&hgta_ doSchema $\mathrm{Db}=$ hg19\&hgta_doSchemaTable=tfbsConsFactors) at the UCSC Genome Browser (28). These binding sites are conserved across the human/mouse/rat alignment. To further reduce the false-positive prediction, P-value of 0.05 was used as a cut-off for high-quality TFBSs. A gene is identified as the target of a TF if at least one TFBS is located in the gene's promoter region (5,000 bp upstream and 1,000 bp downstream of the TSS) and its P-value was $<0.05$. miRNA overlaps with a known host transcript (mRNA/lncRNA) and serve as a part of the same transcription unit. Therefore, the promoter for this specific transcript is used as the miRNA promoter. We identified the target miRNAs of the TFs by using the same approach as for protein-coding genes and lncRNAs. A miRNA is regarded as the target of a TF if at least one TFBS falls within the TSS region (from 5,000 bp upstream to 1,000 bp downstream) of the host gene or lncRNA and its P-value was $<0.05$.

Combined differentially expressed TFs with TFBS prediction. To further increase the accuracy of TFBS prediction, differentially expressed TFs (fold-change, $\geq 2.0$ ) were overlapped with the predicted TFs above. Subsequently, we extracted TFs and miRNA pairs, TFs and miRNA target genes pairs to form $\mathrm{TF} \rightarrow \mathrm{miRNA}$ and $\mathrm{TF} \rightarrow$ gene regulatory relations, respectively.

miRNA-TF regulatory network construction and network node analysis. The $\mathrm{TF} \rightarrow \mathrm{miRNA}$ and $\mathrm{TF} \rightarrow$ gene interactions were incorporated into miRNA $\rightarrow$ gene interactions to construct a comprehensive miRNA-TF mediated regulatory network, which was visualized using Gephi software (version 0.8.1beta, http:/gephi.github.io/). For further network functional 
A

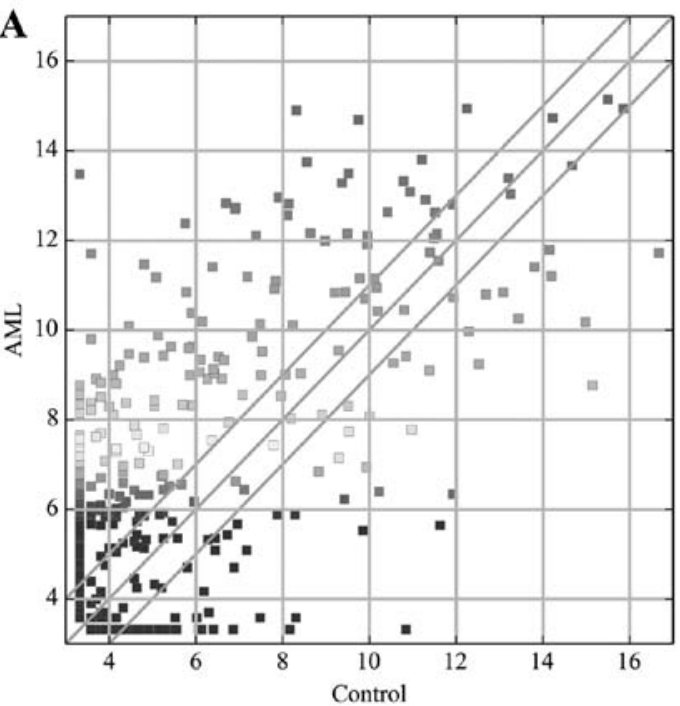

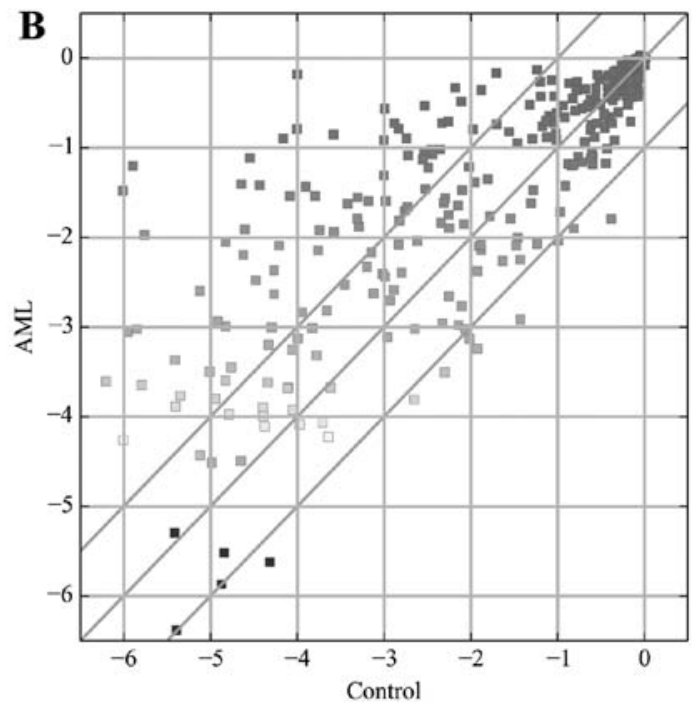

Figure 1. miRNA and TF profile comparison between the bone marrow samples of AML patients and control groups. The scatter-plot is used for evaluating (A) the miRNA and (B) TF expression variation between the bone marrow samples of AML patients and control groups. The values of x-axes and y-axes in the scatter-plot are averaged normalized values for each group (log2 scaled). The oblique lines represent fold-change lines (the cut-off value for fold-change was set to 2.0). The miRNAs and TFs above the top oblique line and below the bottom oblique line indicate $>2$-fold change of miRNAs and TFs between the two sets of bone marrow samples.
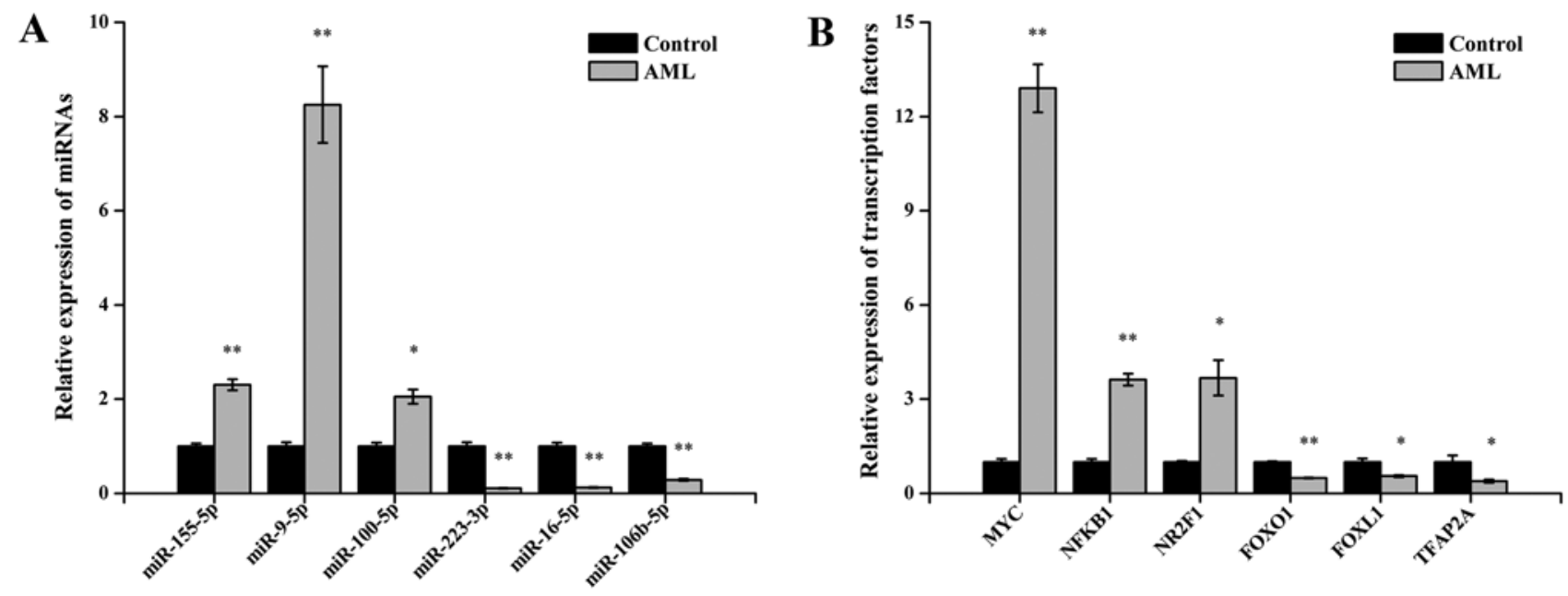

Figure 2. Validation of the high-throughput sequencing and microarray data by qPCR. The relative expression level of each miRNA (A) was normalized to U6 snRNA, and each TF (B) was normalized to $18 \mathrm{~S}$ rRNA. Data in histograms are means $\pm \mathrm{SD} ;{ }^{*} \mathrm{P}<0.05,{ }^{* *} \mathrm{P}<0.01$ comparing AML samples with control samples (Student's t-test).

evaluation, GO analysis and KEGG pathway analysis were performed using the Functional Annotation Tool of DAVID database (version v6.7, http://david.abcc.ncifcrf.gov/) (29) to identify the functional categories enriched and pathways for the network nodes. GO term enrichment and the KEGG pathways utilized significant $\mathrm{P}$-values $(<0.05)$ relating to the nodes of miRNA-TF regulatory network. To assess network characteristics, the node degree is measured by the number of direct links of the node in the network. Nodes having a total degree $\geq 20$ were defined as hub nodes inside the network. The subnetworks were then constructed by combining all the directly linked nodes for the hubs.

Statistical analysis. Statistical analyses were performed are presented as means \pm standard deviation (SD). The using
SPSS for Windows (version 13.0; SPSS, Inc.). All values statistical difference between means were analyzed by the Student's t-test and P-value $<0.05$ was considered significant.

\section{Results}

Aberrant miRNA and TF expression in AML. To obtain AML-related miRNAs and TFs, we analyzed miRNA and TF expression profiles in bone marrow samples of AML patients and control groups, using miRNA sequencing and TF array technology, respectively. Among the miRNA transcripts examined, we found 308 miRNAs were differentially expressed (fold-change $\geq 2.0$ ) in AML samples relative to their controls (Fig. 1A), with 233 being upregulated, while 75 were downregulated. miR-206 (fold-change, 1144) was 


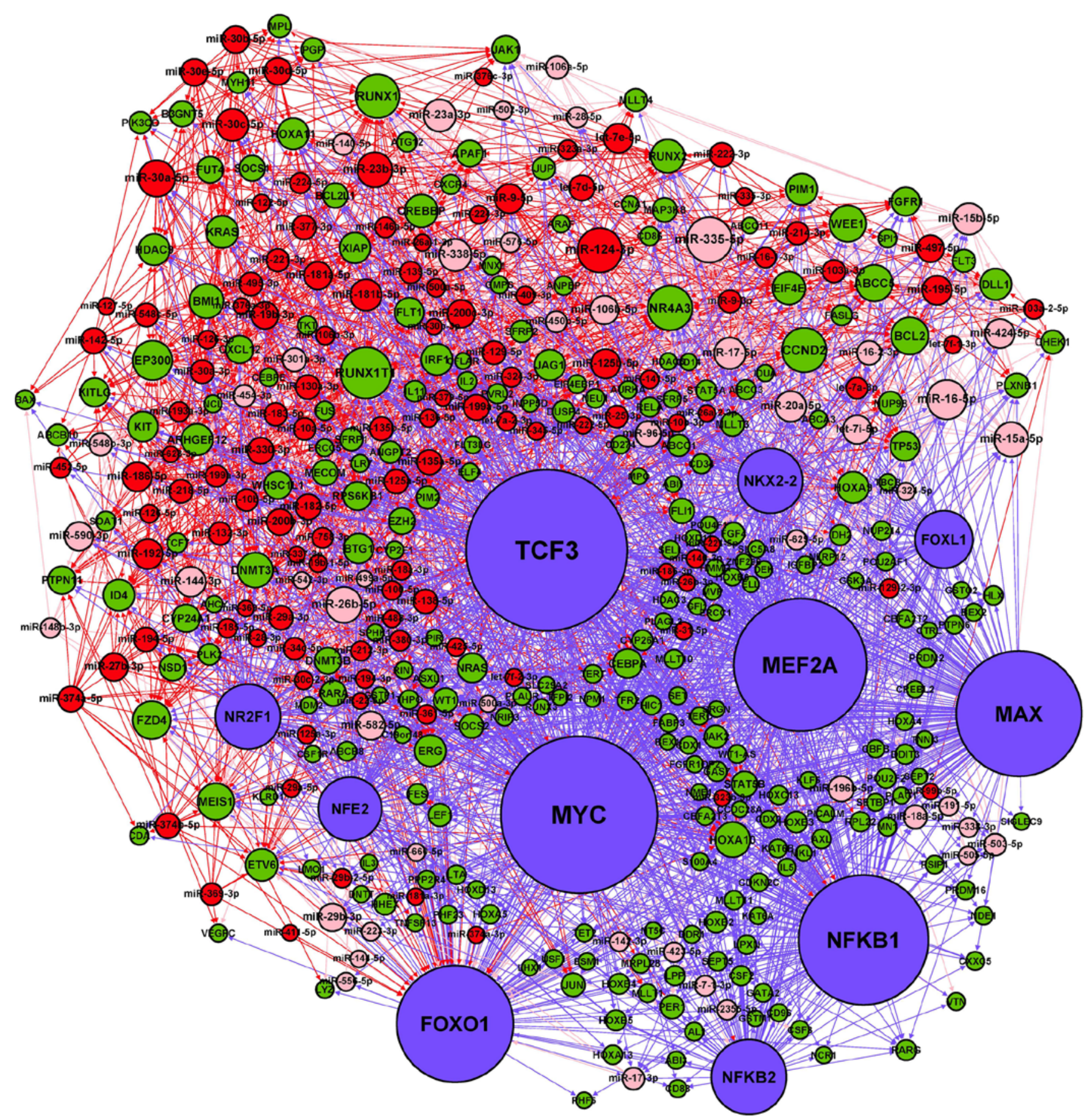

Figure 3. miRNA and TF regulatory network in AML. Red nodes, upregulated AML-related miRNAs; pink nodes, downregulated AML-related miRNAs; green nodes, AML candidate genes; blue nodes, TFs. Red arrows, regulatory relations for upregulated miRNA and target gene; pink arrows, regulatory relations for downregulated miRNA and target gene; blue arrows, regulatory relations for TF-miRNA or TF-gene.

the most significantly upregulated miRNA, while miR-941 (fold-change, 184.2) was the most significantly downregulated miRNA.

The TF expression profiling data showed 84 TFs to be differentially expressed (fold-change $\geq 2.0$ ) in AML samples relative to their controls (Fig. 1B), with 76 upregulated, while 8 were downregulated. Among these TFs, PREB (foldchange, 25.9) showed the highest degree of upregulation, while HiNF-B (fold-change, 2.8) was the most downregulated TF.
Quantitative PCR validation. To validate the miRNA sequencing and TF array results, 6 differentially expressed miRNAs and 6 differentially expressed TFs were selected and analyzed via qPCR. For the miRNAs, the results demonstrated that miR-155-5p, miR-9-5p and miR-100-5p were upregulated and that miR-223-3p, miR-16-5p and miR-106b-5p were downregulated in the AML samples compared with control samples $(\mathrm{P}<0.05$ for each miRNAs; Fig. 2A). For the TFs, the expression of MYC, NFKB1, NR2F1, FOXO1, FOXL1 and TFAP2A showed statistically significant differences between the two 

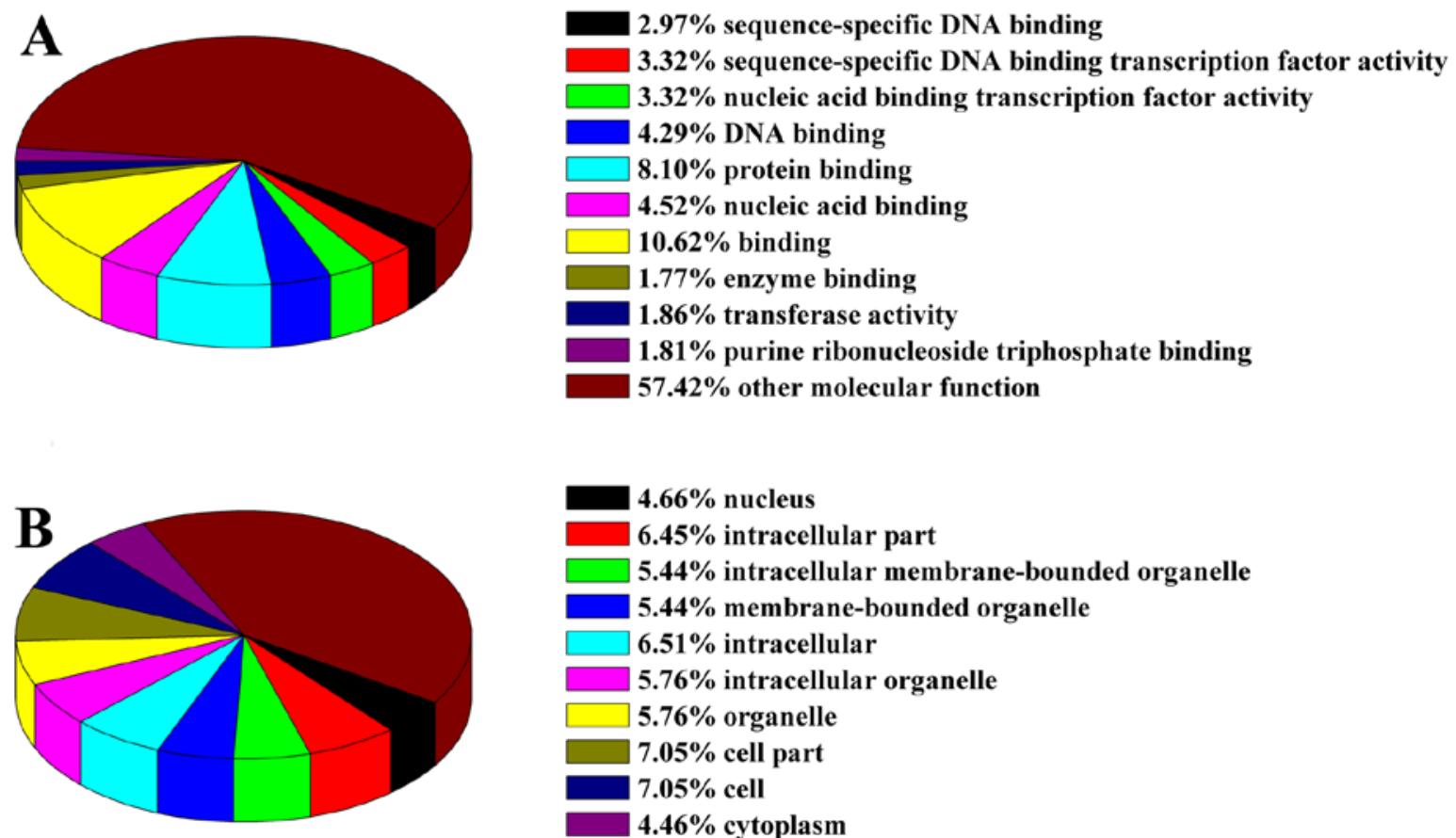

$4.66 \%$ nucleus

$6.45 \%$ intracellular part

$5.44 \%$ intracellular membrane-bounded organelle

$5.44 \%$ membrane-bounded organelle

$6.51 \%$ intracellular

$5.76 \%$ intracellular organelle

$\mathbf{5 . 7 6} \%$ organelle

$7.05 \%$ cell part

$7.05 \%$ cell

$4.46 \%$ cytoplasm

$41.42 \%$ other cellular component

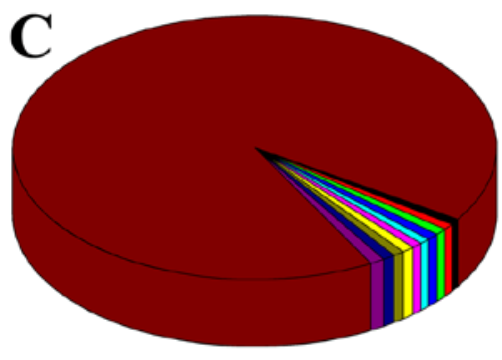

$0.68 \%$ multicellular organismal process

$0.78 \%$ regulation of cellular process

$0.80 \%$ regulation of biological process

$0.82 \%$ biological regulation

$0.69 \%$ cellular macromolecule metabolic process

$0.73 \%$ macromolecule metabolic process

$0.79 \%$ cellular metabolic process

$\mathbf{0 . 7 9} \%$ primary metabolic process

$0.82 \%$ metabolic process

$0.95 \%$ cellular process

$92.15 \%$ other biological process

Figure 4. Gene Ontology (GO) enrichment analysis for nodes in the miRNA-TF regulatory network. GO analysis was performed to determine the enrichment of network nodes in molecular functions (A), cellular components (B), and biological processes (C). A P-value of $<0.05$ was considered significant.

sets of bone marrow samples (all $\mathrm{P}<0.05$; Fig. 2B). These qPCR results were consistent with the miRNA sequencing and TF array data.

miRNA and TF regulatory network in AML. The basic integrated regulatory network for miRNA and TF consists of three types of interactions: miRNA $\rightarrow$ gene (including miRNA $\rightarrow \mathrm{TF}$ ), $\mathrm{TF} \rightarrow \mathrm{miRNA}$ and $\mathrm{TF} \rightarrow$ gene regulatory relations. After removing a few isolated nodes, we merged the three regulatory relations and constructed a miRNA-TF regulatory network for AML (Fig. 3). The numbers of nodes and pairs in the network are listed in Table V. Among the AML-related miRNAs in the network, most of (117/173) them are upregulated. Moreover, there were three pairs of miRNA $\rightarrow$ gene regulatory relationships (miR-126-5p and miR-126-3p represses PLK2 and miR-17-5p represses RUNX1) that have been experimentally confirmed in AML $(30,31)$. Two TFs (NFKB1 and MYC) in this network have been reportedly associated with the development of AML $(32,33)$. NFKB1 and MYC transcriptionally suppressed miR-29b-3p expression by binding to its promoter, and MYC was confirmed as a transcriptional repressor of miR-15a-5p,
Table V. Summary of relationships in the AML-related miRNA and transcription factor (TF) regulatory network.

\begin{tabular}{lrccc}
\hline Relationship & $\begin{array}{c}\text { No. of } \\
\text { pairs }\end{array}$ & $\begin{array}{c}\text { No. of } \\
\text { miRNAs }\end{array}$ & $\begin{array}{c}\text { No. of } \\
\text { genes }\end{array}$ & $\begin{array}{c}\text { No. of } \\
\text { TFs. }\end{array}$ \\
\hline miRNA-gene $^{\mathrm{a}}$ & 1462 & 173 & 150 & - \\
miRNA-TF $^{\mathrm{b}}$ & 64 & 49 & - & 5 \\
TF-gene $^{\mathrm{c}}$ & 982 & - & 264 & 10 \\
TF-miRNA $^{\mathrm{d}}$ & 296 & 114 & - & 11 \\
\hline
\end{tabular}

${ }^{\mathrm{a}}$ miRNA repression of gene expression. ${ }^{\mathrm{b}}$ miRNA repression of $\mathrm{TF}$ expression. ${ }^{\mathrm{T}} \mathrm{TF}$ regulation of gene expression. ${ }^{\mathrm{d}} \mathrm{TF}$ regulation of miRNA expression.

which were in accord with the same $\mathrm{TF} \rightarrow \mathrm{miRNA}$ regulatory relationships in our network $(34,35)$. As key regulators of gene expression, miRNAs and TFs may reciprocally regulate each other to form FBLs, or co-regulate the expression of the 
Table VI. Pathway analysis for network nodes in AML-related miRNA and transcription factor regulatory network.

\begin{tabular}{|c|c|c|c|c|}
\hline Pathway ID & Definition & Gene count & $\%$ & Fisher-P-value \\
\hline hsa05221 & Acute myeloid leukemia & 24 & 9.09 & $1.31 \mathrm{E}-21$ \\
\hline hsa05200 & Pathways in cancer & 43 & 16.29 & $5.77 \mathrm{E}-18$ \\
\hline hsa04630 & Jak-STAT signaling pathway & 22 & 8.33 & $1.21 \mathrm{E}-09$ \\
\hline hsa05220 & Chronic myeloid leukemia & 15 & 5.68 & $1.32 \mathrm{E}-08$ \\
\hline hsa05215 & Prostate cancer & 16 & 6.06 & $1.67 \mathrm{E}-08$ \\
\hline hsa04640 & Hematopoietic cell lineage & 15 & 5.68 & $8.18 \mathrm{E}-08$ \\
\hline hsa04210 & Apoptosis & 12 & 4.55 & $2.65 \mathrm{E}-05$ \\
\hline hsa05210 & Colorectal cancer & 11 & 4.17 & $1.05 \mathrm{E}-04$ \\
\hline hsa04060 & Cytokine-cytokine receptor interaction & 19 & 7.20 & $3.44 \mathrm{E}-04$ \\
\hline hsa05222 & Small cell lung cancer & 10 & 3.79 & $5.23 \mathrm{E}-04$ \\
\hline hsa05213 & Endometrial cancer & 8 & 3.03 & $5.57 \mathrm{E}-04$ \\
\hline hsa04012 & ErbB signaling pathway & 10 & 3.79 & $6.78 \mathrm{E}-04$ \\
\hline hsa05211 & Renal cell carcinoma & 9 & 3.41 & $6.95 \mathrm{E}-04$ \\
\hline hsa04660 & $\mathrm{T}$ cell receptor signaling pathway & 11 & 4.17 & 8.30E-04 \\
\hline hsa05212 & Pancreatic cancer & 9 & 3.41 & $8.41 \mathrm{E}-04$ \\
\hline hsa05219 & Bladder cancer & 7 & 2.65 & 0.001013 \\
\hline hsa05216 & Thyroid cancer & 6 & 2.27 & 0.001129 \\
\hline hsa02010 & $\mathrm{ABC}$ transporters & 7 & 2.65 & 0.001302 \\
\hline hsa04722 & Neurotrophin signaling pathway & 11 & 4.17 & 0.002384 \\
\hline hsa04010 & MAPK signaling pathway & 17 & 6.44 & 0.003123 \\
\hline hsa04310 & Wnt signaling pathway & 12 & 4.54 & 0.003263 \\
\hline hsa05218 & Melanoma & 8 & 3.03 & 0.00355 \\
\hline hsa04910 & Insulin signaling pathway & 11 & 4.17 & 0.004425 \\
\hline hsa04662 & B cell receptor signaling pathway & 8 & 3.03 & 0.004828 \\
\hline hsa04520 & Adherens junction & 8 & 3.03 & 0.005583 \\
\hline hsa04916 & Melanogenesis & 9 & 3.41 & 0.006365 \\
\hline hsa04110 & Cell cycle & 10 & 3.79 & 0.008204 \\
\hline hsa04672 & Intestinal immune network for IgA production & 6 & 2.27 & 0.01157 \\
\hline hsa04650 & Natural killer cell mediated cytotoxicity & 10 & 3.79 & 0.012086 \\
\hline hsa04664 & Fc epsilon RI signaling pathway & 7 & 2.65 & 0.021766 \\
\hline hsa04620 & Toll-like receptor signaling pathway & 8 & 3.03 & 0.022915 \\
\hline hsa05214 & Glioma & 6 & 2.27 & 0.031252 \\
\hline hsa04115 & p53 signaling pathway & 6 & 2.27 & 0.04151 \\
\hline
\end{tabular}

same targets to form FFLs (18). In our miRNA-TF regulatory network, we identified $13 \mathrm{FBL}$ and $1156 \mathrm{FFL}$ motifs, reflecting the tight relationships between miRNAs and TFs in the network.

Biological functions of the miRNA-TF regulatory network. To investigate the functional groupings of the miRNA-TF regulatory network in AML, we applied the DAVID database to classify the distinct network nodes according to their GO molecular function, cellular component and biological process terms (Fig. 4). In the GO molecular function classification, a large proportion of nodes associated with binding, protein binding and nucleic acid binding (Fig. 4A). The nodes related to cellular components were mainly located in the cell parts, cell and intracellular (Fig. 4B). The GO biological processes classification showed that nodes were mainly involved in cellular process, metabolic process and biological regulation (Fig. 4C). To ascertain which pathways are enriched in the network nodes, KEGG pathway analysis was also performed by using the DAVID database. The results indicate that the nodes were significantly enriched in 33 different pathways, of which the AML pathway was the most significant followed by the pathways in cancer and Jak-STAT signaling pathways (Table VI). Among these pathways, 17 were related to hematopoiesis and leukemia. In addition, AML pathway, apoptosis pathway, Jak-STAT signaling pathway, MAPK signaling pathway, Wnt signaling pathway, p53 signaling pathway, cell cycle pathway and ABC transporter pathway have been previously experimentally verified to be involved in AML (2,36-42).

Network hubs and subnetworks for hubs in the miRNA-TF regulatory network. To ascertain which nodes potentially have the most influence on the overall behavior of the networks, 
Table VII. Hub miRNAs in AML-related miRNA and transcription factor regulatory network.

\begin{tabular}{|c|c|c|c|c|}
\hline Top & miRNA & In-degree & Out-degree & Total degree \\
\hline 1 & $\operatorname{miR}-335-5 p$ & 1 & 41 & 42 \\
\hline 2 & miR-124-3p & 0 & 41 & 41 \\
\hline 3 & miR-16-5p & 4 & 30 & 34 \\
\hline 4 & miR-30a-5p & 4 & 26 & 30 \\
\hline 5 & $\operatorname{miR}-26 b-5 p$ & 1 & 28 & 29 \\
\hline 6 & $\operatorname{miR}-23 b-3 p$ & 0 & 28 & 28 \\
\hline 7 & miR-15a-5p & 6 & 21 & 27 \\
\hline 8 & $\operatorname{miR}-23 a-3 p$ & 0 & 24 & 24 \\
\hline 9 & $\operatorname{miR}-338-5 p$ & 3 & 21 & 24 \\
\hline 10 & $\operatorname{miR}-30 c-5 p$ & 4 & 20 & 24 \\
\hline 11 & miR-15b-5p & 4 & 19 & 23 \\
\hline 12 & miR-17-5p & 7 & 16 & 23 \\
\hline 13 & miR-181b-5p & 4 & 19 & 23 \\
\hline 14 & miR-20a-5p & 7 & 15 & 22 \\
\hline 15 & miR-144-3p & 0 & 21 & 21 \\
\hline 16 & miR-192-5p & 3 & 18 & 21 \\
\hline 17 & miR-424-5p & 5 & 15 & 20 \\
\hline 18 & let-7e-5p & 0 & 20 & 20 \\
\hline 19 & $\operatorname{miR}-125 b-5 p$ & 3 & 17 & 20 \\
\hline 20 & miR-186-5p & 1 & 19 & 20 \\
\hline 21 & miR-195-5p & 3 & 17 & 20 \\
\hline 22 & $\operatorname{miR}-9-5 p$ & 1 & 19 & 20 \\
\hline
\end{tabular}

we identified the hub nodes based on the highest total degrees inside the network. As shown in Tables VII and VIII, 22 miRNAs and 11 TFs were defined as hub nodes, which having a total degree $\geq 20$. It is of interest that five of the 22 hub miRNAs belonged to the miR-15 family, namely miR-15a-5p/ miR-15b-5p/miR-16-5p/miR-424-5p/miR-195-5p (Table VII), further indicating the important role of the miR-15 family in the regulatory network of AML. Notably, more than half of the 11 hub TFs were either notorious leukemia regulators, such as NFE2 (43) and MYC (33), or related to leukemia development and progression, such as NFKB1 (32), TCF3 (44), MAX (45) and FOXO1 (46). These findings are a preliminary reflection on the robustness of our network. Among these hub nodes, two hub miRNAs (miR-15a-5p and miR-125b-5p) and two hub TFs (MYC and NFKB1) have been previously implicated in AML $(12,32,33,47)$. To further investigate the regulation of the four hub nodes, we extracted their subnetworks by including all their directly linked nodes in the miRNA-TF regulatory network (Fig. 5). The subnetwork analysis indicated that many pathways related to AML and other cancers (Table IX), which suggested that miR-15a-5p, miR-125b-5p, MYC and NFKB1 were important in cancer development including AML. In addition, TCF3 stood out as a promising regulator and gene, which links to all the four subnetworks. As a TF, it regulates miR-125b-5p (Fig. 5B) and MYC (Fig. 5C); while in its capacity as a gene, it is regulated by miR-15a-5p (Fig. 5A), MYC (Fig. 5C) and NFKB1 (Fig. 5D). In particular,
Table VIII. Hub transcription factors in AML-related miRNA and transcription factor regulatory network.

\begin{tabular}{ccrcc}
\hline Top & $\begin{array}{c}\text { Transcription } \\
\text { factor }\end{array}$ & In-degree & Out-degree & $\begin{array}{c}\text { Total } \\
\text { degree }\end{array}$ \\
\hline 1 & TCF3 & 14 & 192 & 206 \\
2 & MYC & 9 & 186 & 195 \\
3 & MEF2A & 0 & 167 & 167 \\
4 & NFKB1 & 8 & 153 & 161 \\
5 & MAX & 0 & 152 & 152 \\
6 & FOXO1 & 43 & 98 & 141 \\
7 & NFKB2 & 3 & 80 & 83 \\
8 & NFE2 & 0 & 73 & 73 \\
9 & NR2F1 & 0 & 73 & 73 \\
10 & NKX2-2 & 0 & 71 & 71 \\
11 & FOXL1 & 0 & 59 & 59 \\
\hline
\end{tabular}

17 predicted target genes (CEBPA, FLT3, STAT5A, RELA, STAT5B, PIM1, SPI1, RUNX1T1, LEF1, PIM2, KIT, JUP, NRAS, EIF4EBP1, KRAS, RARA and MYC) of TCF3 in the four subnetworks participated in the AML pathway. These analyses suggest that TCF3 may play an important role in the miRNA-TF regulatory network linked to AML.

\section{Discussion}

AML is the most common form of aggressive leukemia in adults $(1,2)$. Despite extensive efforts to elucidate the cytogenetic and molecular genetic mechanisms involved in disease occurrence and development, the pathogenesis of AML is still not fully understood due to the heterogeneity and complexity of this disease. The genomic complexity makes it difficult to identify hub regulators or genes in the pathogenesis of AML. By integrating diverse data sources, systems biology approaches provide a powerful tool for exploring the interactions and searching for key regulators between disease candidate genes on the network level, which may help to explain key aspects of disease pathogenesis and leading to new candidates for the putative therapeutic targets (48).

As major regulators of gene expression, miRNAs and TFs can function as tumor suppressors or oncogenes in a cooperative way to control gene expression, which triggering global alterations of genetic programs are involved in cell proliferation, differentiation, development and apoptosis in multiple human cancers (18). Ye et al (49) constructed a miRNA-TF regulatory network in T-cell acute lymphoblastic leukemia and demonstrated the roles of miR-19 (hub miRNA) and CYLD (hub gene) in the T-cell leukemogenesis. Therefore, understanding and applying regulatory network information for TF-miRNA-target genes could provide clues for key driver miRNAs and genes in human cancers and subsequently suggest novel therapeutic targets (50).

In the present study, we applied miRNA sequencing and TF array technology to quantitatively analyze the differential expression profiles of miRNAs and TFs, respectively, in bone 

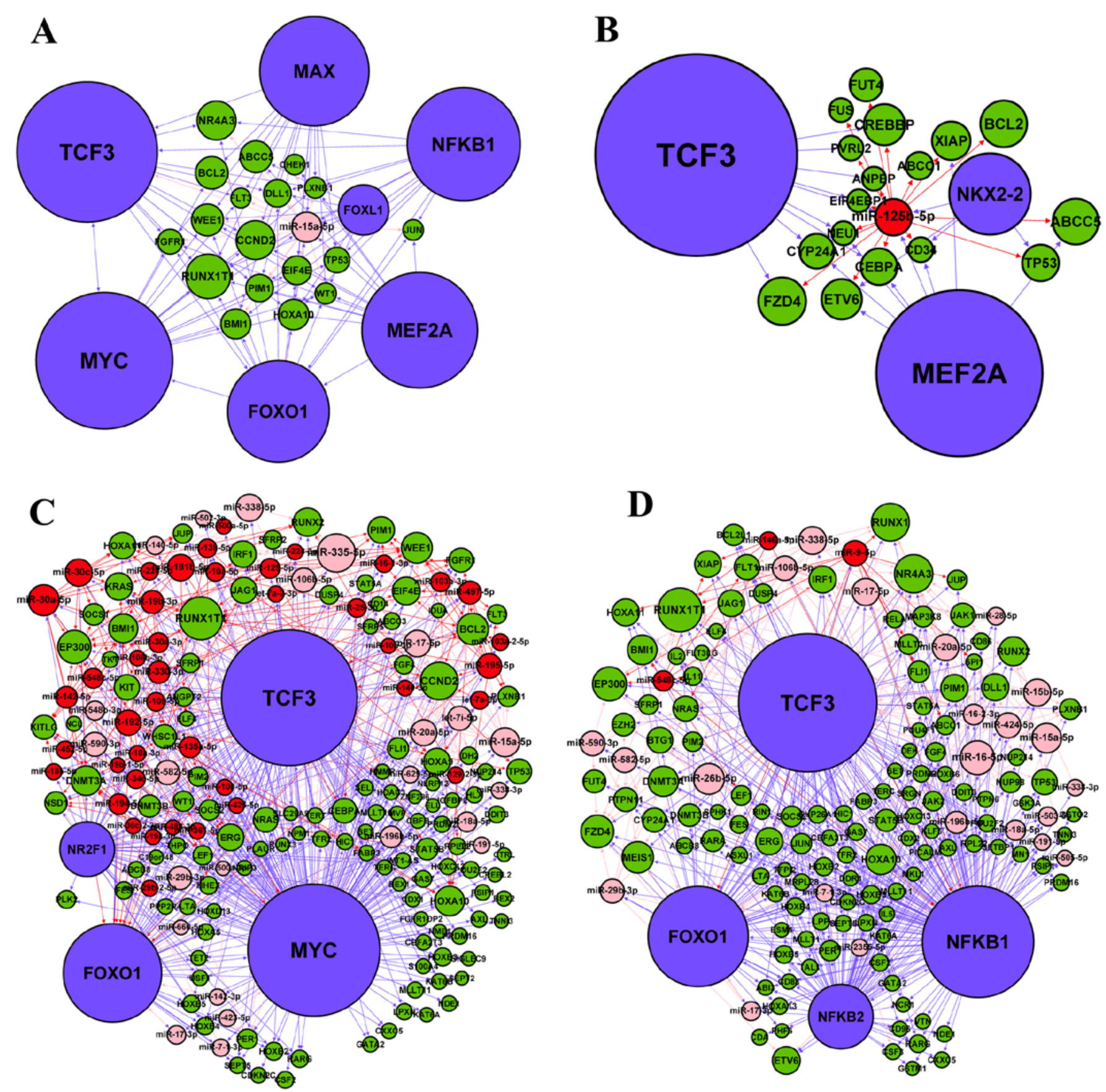

Figure 5. Subnetworks of the four hub nodes in our miRNA-TF regulatory network. The subnetworks were drawn by all the directly linked nodes for the hubs. (A) miR-15a-5p; (B) miR-125b-5p; (C) MYC; (D) NFKB1. Red nodes, upregulated AML-related miRNAs; pink nodes, downregulated AML-related miRNAs; green nodes, AML candidate genes; blue nodes, TFs. Red arrows, regulatory relations for upregulated miRNA and target gene; pink arrows, regulatory relations for downregulated miRNA and target gene; blue arrows, regulatory relations for TF-miRNA or TF-gene.

marrow samples of AML patients and their age-matched controls (Fig. 1). The expression profiling data showed that 308 miRNAs and 84 TFs were differentially expressed (foldchange $\geq 2.0$ ) in AML samples relative to their controls, with a subset of these findings further validated by qPCR (Fig. 2). It is difficult to achieve by only direct experimental methods to systematically infer the comprehensive miRNA-TF regulatory network, which has promoted development of computational approaches. Therefore, we integrated expression profiling data of miRNAs and TFs, computational miRNA and TF target prediction, and AML candidate genes obtained from
MalaCards database to construct a miRNA and TF regulatory network specifically for AML (Fig. 3), which might provide important insights into the pathogenesis of AML. Moreover, we found that some miRNA $\rightarrow$ gene and $\mathrm{TF} \rightarrow$ miRNA regulatory relationships in our network have been experimentally verified in previous studies. For further network functional evaluation, GO category and KEGG pathway annotation were applied to analyze the network node pool (Fig. 4 and Table VI). Among 33 enriched pathways, the AML pathway was the most significant (Table VI), more than half of these pathways were related to hematopoiesis and leukemia development, and eight pathways 
Table IX. Pathways enriched among the nodes of four subnetworks.

\begin{tabular}{|c|c|c|}
\hline Subnetwork & $\begin{array}{l}\text { Enriched pathway } \\
\text { of subnetwork nodes }\end{array}$ & P-value \\
\hline \multirow[t]{12}{*}{$\operatorname{miR}-15 a-5 p$} & Pathways in cancer & $2.66 \mathrm{E}-07$ \\
\hline & Acute myeloid leukemia & $3.25 \mathrm{E}-05$ \\
\hline & Small cell lung cancer & $1.40 \mathrm{E}-04$ \\
\hline & Prostate cancer & $1.75 \mathrm{E}-04$ \\
\hline & Cell cycle & $6.46 \mathrm{E}-04$ \\
\hline & MAPK signaling pathway & 0.001412 \\
\hline & Colorectal cancer & 0.002502 \\
\hline & Neurotrophin signaling pathway & 0.007495 \\
\hline & Wnt signaling pathway & 0.012855 \\
\hline & p53 signaling pathway & 0.021056 \\
\hline & Chronic myeloid leukemia & 0.025301 \\
\hline & Apoptosis & 0.033319 \\
\hline \multirow[t]{5}{*}{$\operatorname{miR}-125 b-5 p$} & Pathways in cancer & 0.001898 \\
\hline & Colorectal cancer & 0.024629 \\
\hline & Small cell lung cancer & 0.024629 \\
\hline & Apoptosis & 0.026297 \\
\hline & Prostate cancer & 0.027434 \\
\hline \multirow[t]{17}{*}{ MYC } & Acute myeloid leukemia & $5.58 \mathrm{E}-13$ \\
\hline & Pathways in cancer & $3.73 \mathrm{E}-08$ \\
\hline & Prostate cancer & $4.59 \mathrm{E}-05$ \\
\hline & Jak-STAT signaling pathway & $2.55 \mathrm{E}-04$ \\
\hline & Thyroid cancer & $2.55 \mathrm{E}-04$ \\
\hline & Wnt signaling pathway & 0.001212 \\
\hline & Chronic myeloid leukemia & 0.001297 \\
\hline & Hematopoietic cell lineage & 0.002388 \\
\hline & Endometrial cancer & 0.002413 \\
\hline & Melanogenesis & 0.004401 \\
\hline & Melanoma & 0.007421 \\
\hline & MAPK signaling pathway & 0.008221 \\
\hline & Bladder cancer & 0.010721 \\
\hline & Cell cycle & 0.011628 \\
\hline & Colorectal cancer & 0.013264 \\
\hline & ErbB signaling pathway & 0.014936 \\
\hline & Insulin signaling pathway & 0.015823 \\
\hline \multirow[t]{12}{*}{ NFKB1 } & Acute myeloid leukemia & $2.05 \mathrm{E}-12$ \\
\hline & Pathways in cancer & $8.66 \mathrm{E}-11$ \\
\hline & Jak-STAT signaling pathway & $2.73 \mathrm{E}-09$ \\
\hline & Chronic myeloid leukemia & 2.73E-06 \\
\hline & $\mathrm{T}$ cell receptor signaling pathway & 4.17E-05 \\
\hline & Prostate cancer & $6.79 \mathrm{E}-04$ \\
\hline & MAPK signaling pathway & 0.004636 \\
\hline & Pancreatic cancer & 0.011110 \\
\hline & B cell receptor signaling pathway & 0.012775 \\
\hline & Neurotrophin signaling pathway & 0.016993 \\
\hline & Small cell lung cancer & 0.018703 \\
\hline & Hematopoietic cell lineage & 0.020218 \\
\hline
\end{tabular}

Table IX. Continued.

\begin{tabular}{|c|c|c|}
\hline Subnetwork & $\begin{array}{l}\text { Enriched pathway } \\
\text { of subnetwork nodes }\end{array}$ & P-value \\
\hline \multirow[t]{7}{*}{ NFKB1 } & Apoptosis & 0.021003 \\
\hline & Toll-like receptor signaling pathway & 0.033987 \\
\hline & Wnt signaling pathway & 0.036016 \\
\hline & $\begin{array}{l}\text { Cytokine-cytokine receptor } \\
\text { interaction }\end{array}$ & 0.038854 \\
\hline & Adipocytokine signaling pathway & 0.047655 \\
\hline & Thyroid cancer & 0.048101 \\
\hline & $\begin{array}{l}\text { Epithelial cell signaling in } \\
\text { Helicobacter pylori infection }\end{array}$ & 0.049434 \\
\hline
\end{tabular}

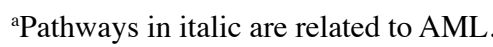

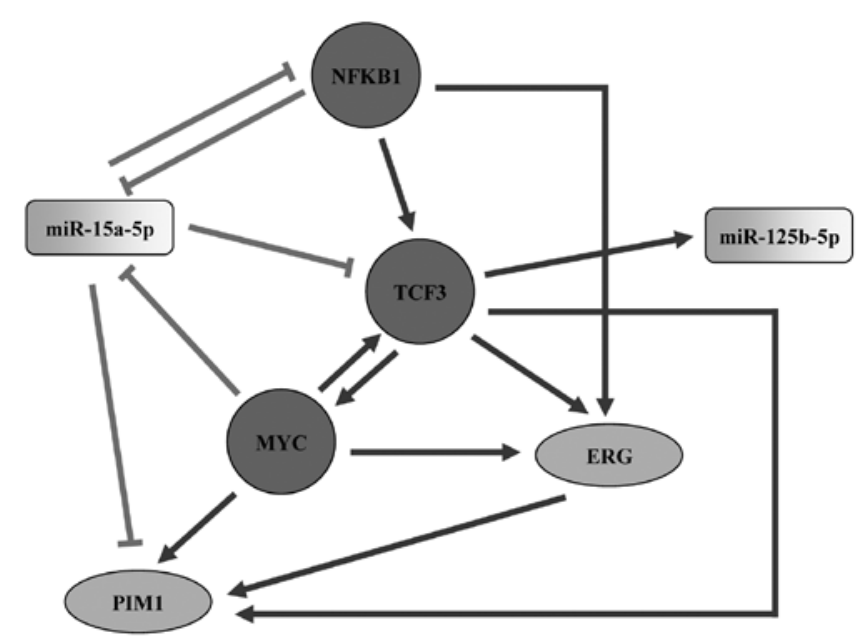

Figure 6. A schematic model of TCF3 and the four hub nodes involved in subnetworks in AML. Round nodes, TFs; rectangle nodes, miRNAs; ellipse nodes, genes. Edges, sharp arrow means activation; T-shaped arrow represents repression.

have been reported to be implicated in AML pathogenesis. These results preliminarily confirmed the reliability of our network.

In the miRNA-TF regulatory network, we identified $22 \mathrm{hub}$ miRNAs and 11 hub TFs. With a large number of nodes linked to them, these hubs have the most influence on the networks overall behavior, which likely play critical regulatory roles in AML. To decipher this massive and complex network, and mine the key regulatory components, we subsequently constructed the subnetworks (Fig. 5) from two hub miRNAs (miR-15a-5p and miR-125b-5p) and two hub TFs (MYC and NFKB1) which were well-known regulators in AML. miR-15a-5p plays an important role in myeloid and erythroid differentiation, which was reported as a tumor suppressor in multiple myeloma, chronic lymphocytic leukemia and AML cells $(51,52)$. Overexpression of miR-15a-5p significantly inhibited AML cell proliferation by downregulating the WT1 oncogene (51). miR-125b-5p is an oncogenic miRNA, which was highly expressed in AML carrying the $t(2 ; 11)(\mathrm{p} 21 ; \mathrm{q} 23)$ 
translocation and inhibited myeloid differentiation (12). Mice overexpressing miR-125b-5p causes a highly aggressive myeloid leukemia related to targeting the gene Lin28A (53). MYC is a basic transcription factor of the helix-loop-helixleucine zipper family, which is found upregulated in AML and has been shown to block the myeloid cell differentiation (33). Retroviral transduction of MYC into murine bone marrow cells results in AML development $(33,54)$. Similarly, sustained expression of a human MYC transgene culminated in the formation of AML in mice (33). NFKB1 is a transcription factor that is constitutively activated in primitive human AML cells, while inhibition of NFKB1 displayed a rapid induction of apoptosis in AML cells $(32,55)$. After analyzing the topology of subnetwork, we found that TCF3 was a potential key regulator in our network due to its direct linking to all the four hubs in the subnetworks (Fig. 5), which may establish cross-talk among these subnetworks. In particular, TCF3 was predicted to regulate 17 target genes which have been involved in AML pathway in the four subnetworks. As a member of the TCF/LEF transcription factor family, TCF3 is essential for development, lymphopoiesis and stem cell function $(44,56)$. Emerging evidence suggests that TCF3 is involved in the pathogenesis of several types of human cancer including colorectal cancer, prostate cancer and breast cancer (57-59). Furthermore, the $\mathrm{t}(1 ; 19)$ translocation between TCF3 and PBX1 genes causes expression of a TCF3-PBX1 fusion protein, and has been identified in B-cell acute lymphoblastic leukemia (B-ALL), which established a potential relationship between TCF3 and the etiology of B-ALL (44). However, the exact molecular mechanism of TCF3 as it relates to AML occurrence and development is still unknown.

In the subnetworks, TCF3 was potentially targeted by miR-15a-5p, MYC and NFKB1, and also targeting miR-125b-5p, MYC, ERG and PIM1 (Fig. 5). ERG is a megakaryocytic oncogene, which is required for normal megakaryopoiesis and play crucial role in establishing definitive hematopoiesis (60). A high expression level of ERG in AML is associated with poor prognosis (61). Transgenic expression of the human ERG causes early progenitor myeloid leukemia in mice similar to human AML, which was mediated by inducing the expression of the oncogenic kinase PIM1 through binding to a novel 3' enhancer (62). PIM1 is a constitutively active serine/threonine protein kinase that normally functions in the proliferation and survival of hematopoietic cells in response to cytokines and growth factors (63). Overexpression of PIM1 has been detected in a range of solid cancers and hematologic malignancies (64). In AML, PIM1 has been suggested to have important roles in cell proliferation, survival, homing and migration, and the prognostic relevance of PIM1 upregulation was also observed in high ERG-expressing AML by the significantly decreased survival of these patients (62). In addition, ERG was the predicted target of MYC, NFKB1 and TCF3, while PIM1 was predicted to be regulated by miR-15a-5p, MYC and TCF3 in our subnetworks (Fig. 5). The above discussion led us to present a model of the involvement of TCF3 and the four hub nodes in subnetworks within AML (Fig. 6). According to the literature surveys and our subnetwork analysis, we propose that TCF3 is a potential key regulator in the miRNA-TF regulatory network linked to AML, which may play its regulatory role via $\mathrm{miR}-15 \mathrm{a}-5 \mathrm{p}, \mathrm{miR}-125 \mathrm{~b}-5 \mathrm{p}$, MYC, NFKB1, ERG or PIM1.

In summary, this is the first study that describes the global expression profiling of miRNAs and TFs relating to AML using miRNA sequencing and TF array technology, respectively. Applying systems biology approaches to integrate the experimental data to multiple types of computational prediction on gene regulation, we constructed a miRNA-TF regulatory network specifically for AML, which may provide some hub regulators and clues for exploring the molecular mechanisms of AML. Furthermore, the present study also provides potential therapeutic targets for AML and proposes directions for further experimental research.

\section{Acknowledgements}

We sincerely thank all donors who participated in this investigation.

\section{References}

1. Conway O'Brien E, Prideaux S and Chevassut T: The epigenetic landscape of acute myeloid leukemia. Adv Hematol 2014: 103175, 2014.

2. Lee HJ, Daver N, Kantarjian HM, Verstovsek S and Ravandi F: The role of JAK pathway dysregulation in the pathogenesis and treatment of acute myeloid leukemia. Clin Cancer Res 19: 327-335, 2013.

3. Zaidi SK, Trombly DJ, Dowdy CR, Lian JB, Stein JL, van Wijnen AJ and Stein GS: Epigenetic mechanisms in leukemia. Adv Biol Regul 52: 369-376, 2012.

4. Jamieson $\mathrm{K}$ and Odenike O: Late-phase investigational approaches for the treatment of relapsed/refractory acute myeloid leukemia. Expert Opin Pharmacother 13: 2171-2187, 2012.

5. Sabnis H, Bradley HL, Bunting ST, Cooper TM and Bunting KD: Capillary nano-immunoassay for Akt $1 / 2 / 3$ and 4EBP1 phosphorylation in acute myeloid leukemia. J Transl Med 12: 166, 2014.

6. Naoe T and Kiyoi H: Gene mutations of acute myeloid leukemia in the genome era. Int J Hematol 97: 165-174, 2013.

7. Larsson CA, Cote $G$ and Quintás-Cardama A: The changing mutational landscape of acute myeloid leukemia and myelodysplastic syndrome. Mol Cancer Res 11: 815-827, 2013.

8. Abdel-Wahab $\mathrm{O}$ and Levine RL: Mutations in epigenetic modifiers in the pathogenesis and therapy of acute myeloid leukemia. Blood 121: 3563-3572, 2013.

9. Yan S, Yim LY, Lu L, Lau CS and Chan VS: MicroRNA regulation in systemic lupus erythematosus pathogenesis. Immune Netw 14: 138-148, 2014.

10. van Rooij E and Kauppinen S: Development of microRNA therapeutics is coming of age. EMBO Mol Med 6: 851-864, 2014.

11. Babashah S, Sadeghizadeh M, Tavirani MR, Farivar S and Soleimani M: Aberrant microRNA expression and its implications in the pathogenesis of leukemias. Cell Oncol (Dordr) 35: 317-334, 2012.

12. Marcucci G, Mrózek K, Radmacher MD, Garzon R and Bloomfield CD: The prognostic and functional role of microRNAs in acute myeloid leukemia. Blood 117: 1121-1129, 2011.

13. Han YC, Park CY, Bhagat G, Zhang J, Wang Y, Fan JB, Liu M, Zou Y, Weissman IL and Gu H: microRNA-29a induces aberrant self-renewal capacity in hematopoietic progenitors, biased myeloid development, and acute myeloid leukemia. J Exp Med 207: 475-489, 2010.

14. Bai J, Guo A, Hong Z and Kuai W: Upregulation of microRNA100 predicts poor prognosis in patients with pediatric acute myeloid leukemia. Onco Targets Ther 5: 213-219, 2012.

15. Wang Z, Hong Z, Gao F and Feng W: Upregulation of microRNA375 is associated with poor prognosis in pediatric acute myeloid leukemia. Mol Cell Biochem 383: 59-65, 2013.

16. Li Y, Gao L, Luo X, Wang L, Gao X, Wang W, Sun J, Dou L, Li J, $\mathrm{Xu}$ C, et al: Epigenetic silencing of microRNA-193a contributes to leukemogenesis in $\mathrm{t}(8 ; 21)$ acute myeloid leukemia by activating the PTEN/PI3K signal pathway. Blood 121: 499-509, 2013. 
17. Hickey CJ, Schwind S, Radomska HS, Dorrance AM, Santhanam R, Mishra A, Wu YZ, Alachkar H, Maharry K, Nicolet $\mathrm{D}$, et al: Lenalidomide-mediated enhanced translation of $\mathrm{C} / \mathrm{EBP} \alpha-\mathrm{p} 30$ protein up-regulates expression of the antileukemic microRNA-181a in acute myeloid leukemia. Blood 121: 159-169, 2013.

18. Zhang HM, Kuang S, Xiong X, Gao T, Liu C and Guo AY: Transcription factor and microRNA co-regulatory loops: Important regulatory motifs in biological processes and diseases. Brief Bioinform 16: 45-58, 2015.

19. Cheng C, Yan KK, Hwang W, Qian J, Bhardwaj N, Rozowsky J, Lu ZJ, Niu W, Alves P, Kato M, et al: Construction and analysis of an integrated regulatory network derived from high-throughput sequencing data. PLoS Comput Biol 7: e1002190, 2011.

20. Pulikkan JA, Dengler V, Peramangalam PS, Peer Zada AA, Müller-Tidow C, Bohlander SK, Tenen DG and Behre G Cell-cycle regulator E2F1 and microRNA-223 comprise an autoregulatory negative feedback loop in acute myeloid leukemia. Blood 115: 1768-1778, 2010.

21. Katzerke C, Madan V, Gerloff D, Bräuer-Hartmann D, Hartmann JU, Wurm AA, Müller-Tidow C, Schnittger S, Tenen DG, Niederwieser D, et al: Transcription factor C/ EBP $\alpha$-induced microRNA-30c inactivates Notch1 during granulopoiesis and is downregulated in acute myeloid leukemia. Blood 122: 2433-2442, 2013

22. Padró T, Ruiz S, Bieker R, Bürger H, Steins M, Kienast J, Büchner T, Berdel WE and Mesters RM: Increased angiogenesis in the bone marrow of patients with acute myeloid leukemia. Blood 95: 2637-2644, 2000.

23. Aurelius J, Martner A, Brune M, Palmqvist L, Hansson M, Hellstrand $\mathrm{K}$ and Thoren FB: Remission maintenance in acute myeloid leukemia: Impact of functional histamine $\mathrm{H} 2$ receptors expressed by leukemic cells. Haematologica 97: 1904-1908, 2012.

24. Pfaffl MW: A new mathematical model for relative quantification in real-time RT-PCR. Nucleic Acids Res 29: e45, 2001.

25. Sui W, Lin H, Peng W, Huang Y, Chen J, Zhang Y and Dai Y: Molecular dysfunctions in acute rejection after renal transplantation revealed by integrated analysis of transcription factor, microRNA and long noncoding RNA. Genomics 102: 310-322, 2013.

26. Lin Y, Wu J, Chen H, Mao Y, Liu Y, Mao Q, Yang K, Zheng X and Xie L: Cyclin-dependent kinase 4 is a novel target in micoRNA195-mediated cell cycle arrest in bladder cancer cells. FEBS Let 586: 442-447, 2012

27. Rappaport N, Twik M, Nativ N, Stelzer G, Bahir I, Stein TI, Safran M and Lancet D: MalaCards: A comprehensive automatically-mined database of human diseases. Curr Protoc Bioinformatics 47: 1.24.1-1.24.19,2014. doi: 10.1002/0471250953. bi0124s47.

28. Felice B, Cattoglio C, Cittaro D, Testa A, Miccio A, Ferrari G, Luzi L, Recchia A and Mavilio F: Transcription factor binding sites are genetic determinants of retroviral integration in the human genome. PLoS One 4: e4571, 2009.

29. Huang DW, Sherman BT, Tan Q, Kir J, Liu D, Bryant D, Guo Y, Stephens R, Baseler MW, Lane HC, et al: DAVID Bioinformatics Resources: Expanded annotation database and novel algorithms to better extract biology from large gene lists. Nucleic Acids Res 35 (Web Server): W169-W175, 2007

30. Li Z, Lu J, Sun M, Mi S, Zhang H, Luo RT, Chen P, Wang Y, Yan M, Qian Z, et al: Distinct microRNA expression profiles in acute myeloid leukemia with common translocations. Proc Natl Acad Sci USA 105: 15535-15540, 2008

31. Fischer J, Rossetti S, Datta A, Eng K, Beghini A and Sacchi N: miR-17 deregulates a core RUNX1-miRNA mechanism of CBF acute myeloid leukemia. Mol Cancer 14: 7, 2015.

32. Grosjean-Raillard J, Adès L, Boehrer S, Tailler M, Fabre C, Braun T, De Botton S, Israel A, Fenaux P and Kroemer G: Flt3 receptor inhibition reduces constitutive NFkappaB activation in high-risk myelodysplastic syndrome and acute myeloid leukemia. Apoptosis 13: 1148-1161, 2008.

33. Brondfield S, Umesh S, Corella A, Zuber J, Rappaport AR, Gaillard C, Lowe SW, Goga A and Kogan SC: Direct and indirect targeting of MYC to treat acute myeloid leukemia. Cancer Chemother Pharmacol 76: 35-46, 2015.

34. Mott JL, Kurita S, Cazanave SC, Bronk SF, Werneburg NW and Fernandez-Zapico ME: Transcriptional suppression of mir-29b-1/ mir-29a promoter by c-Myc, hedgehog, and NF-kappaB. J Cell Biochem 110: 1155-1164, 2010
35. Tao J, Zhao X and Tao J: c-MYC-miRNA circuitry: A central regulator of aggressive B-cell malignancies. Cell Cycle 13: 191-198, 2014.

36. Steffen B, Müller-Tidow C, Schwäble J, Berdel WE and Serve H: The molecular pathogenesis of acute myeloid leukemia. Crit Rev Oncol Hematol 56: 195-221, 2005.

37. Schimmer AD: Novel therapies targeting the apoptosis pathway for the treatment of acute myeloid leukemia. Curr Treat Options Oncol 8: 277-286, 2007.

38. Kim HS, Lim GY, Hwang J, Ryoo ZY, Huh TL and Lee S Induction of apoptosis by obovatol as a novel therapeutic strategy for acute myeloid leukemia. Int J Mol Med 34: 1675-1680, 2014.

39. Mikesch JH, Steffen B, Berdel WE, Serve H and Müller-Tidow C: The emerging role of Wnt signaling in the pathogenesis of acute myeloid leukemia. Leukemia 21: 1638-1647, 2007.

40. Li L, Tan Y, Chen X, Xu Z, Yang S, Ren F, Guo H, Wang X, Chen Y, Li G, et al: MDM4 overexpressed in acute myeloid leukemia patients with complex karyotype and wild-type TP53. PLoS One 9: e113088, 2014.

41. Chim CS, Wong AS and Kwong YL: Epigenetic inactivation of INK4/CDK/RB cell cycle pathway in acute leukemias. Ann Hematol 82: 738-742, 2003

42. Fukuda Y, Lian S and Schuetz JD: Leukemia and ABC transporters. Adv Cancer Res 125: 171-196, 2015.

43. Li YJ, Higgins RR, Pak BJ, Shivdasani RA, Ney PA, Archer M and Ben-David Y: $\mathrm{p}^{45^{\mathrm{NFE}}}$ is a negative regulator of erythroid proliferation which contributes to the progression of friend virusinduced erythroleukemias. Mol Cell Biol 21: 73-80, 2001.

44. Somasundaram R, Prasad MA, Ungerbäck J and Sigvardsson M: Transcription factor networks in B-cell differentiation link development to acute lymphoid leukemia. Blood 126: 144-152, 2015.

45. Zada AA, Pulikkan JA, Bararia D, Geletu M, Trivedi AK, Balkhi MY, Hiddemann WD, Tenen DG, Behre HM and Behre G: Proteomic discovery of Max as a novel interacting partner of C/EBPalpha: A Myc/Max/Mad link. Leukemia 20: 2137-2146, 2006.

46. Pellicano F, Scott MT, Helgason GV, Hopcroft LE, Allan EK, Aspinall-O'Dea M, Copland M, Pierce A, Huntly BJ, Whetton AD, et al: The antiproliferative activity of kinase inhibitors in chronic myeloid leukemia cells is mediated by FOXO transcription factors. Stem Cells 32: 2324-2337, 2014

47. Gao SM, Xing CY, Chen CQ, Lin SS, Dong PH and Yu FJ: miR-15a and miR-16-1 inhibit the proliferation of leukemic cells by down-regulating WT1 protein level. J Exp Clin Cancer Res 30: 110,2011

48. Cho JH, Gelinas R, Wang K, Etheridge A, Piper MG, Batte K, Dakhallah D, Price J, Bornman D, Zhang S, et al: Systems biology of interstitial lung diseases: Integration of mRNA and microRNA expression changes. BMC Med Genomics 4: 8 , 2011.

49. Ye H, Liu X, Lv M, Wu Y, Kuang S, Gong J, Yuan P, Zhong Z, Li Q, Jia H, et al: MicroRNA and transcription factor co-regulatory network analysis reveals miR-19 inhibits CYLD in T-cell acute lymphoblastic leukemia. Nucleic Acids Res 40: 5201-5214, 2012.

50. Yan Z, Shah PK, Amin SB, Samur MK, Huang N, Wang X, Misra V, Ji H, Gabuzda D and Li C: Integrative analysis of gene and miRNA expression profiles with transcription factor-miRNA feed-forward loops identifies regulators in human cancers. Nucleic Acids Res 40: e135, 2012.

51. Gao SM, Xing CY, Chen CQ, Lin SS, Dong PH and Yu FJ: miR-15a and miR-16-1 inhibit the proliferation of leukemic cells by down-regulating WT1 protein level. J Exp Clin Cancer Res 30: 110, 2011 .

52. Aqeilan RI, Calin GA and Croce CM: miR-15a and miR-16-1 in cancer: Discovery, function and future perspectives. Cell Death Differ 17: 215-220, 2010.

53. Chaudhuri AA, So AY, Mehta A, Minisandram A, Sinha N, Jonsson VD, Rao DS, O'Connell RM and Baltimore D: Oncomir miR-125b regulates hematopoiesis by targeting the gene Lin28A. Proc Natl Acad Sci USA 109: 4233-4238, 2012.

54. Luo H, Li Q, O'Neal J, Kreisel F, Le Beau MM and Tomasson MH: c-Myc rapidly induces acute myeloid leukemia in mice without evidence of lymphoma-associated antiapoptotic mutations. Blood 106: 2452-2461, 2005 
55. Guzman ML, Neering SJ, Upchurch D, Grimes B, Howard DS Rizzieri DA, Luger SM and Jordan CT: Nuclear factor-kappaB is constitutively activated in primitive human acute myelogenous leukemia cells. Blood 98: 2301-2307, 2001.

56. Howard JM, Nuguid JM, Ngole D and Nguyen H: Tcf3 expression marks both stem and progenitor cells in multiple epithelia. Development 141: 3143-3152, 2014.

57. Slyper M, Shahar A, Bar-Ziv A, Granit RZ, Hamburger T, Maly B, Peretz T and Ben-Porath I: Control of breast cancer growth and initiation by the stem cell-associated transcription factor TCF3. Cancer Res 72: 5613-5624, 2012.

58. Li C, Cai S, Wang X and Jiang Z: Hypomethylation-associated up-regulation of TCF3 expression and recurrence in stage II and III colorectal cancer. PLoS One 9: e112005, 2014.

59. Patel D and Chaudhary J: Increased expression of bHLH transcription factor E2A (TCF3) in prostate cancer promotes proliferation and confers resistance to doxorubicin induced apoptosis. Biochem Biophys Res Commun 422: 146-151, 2012.

60. Salek-Ardakani S, Smooha G, de Boer J, Sebire NJ, Morrow M, Rainis L, Lee S, Williams O, Izraeli S and Brady HJ: ERG is a megakaryocytic oncogene. Cancer Res 69: 4665-4673, 2009
61. Marcucci G, Maharry K, Whitman SP, Vukosavljevic T, Paschka P, Langer C, Mrózek K, Baldus CD, Carroll AJ, Powell BL, et al; Cancer and Leukemia Group B Study: High expression levels of the ETS-related gene, ERG, predict adverse outcome and improve molecular risk-based classification of cytogenetically normal acute myeloid leukemia: A Cancer and Leukemia Group B Study. J Clin Oncol 25: 3337-3343, 2007.

62. Goldberg L, Tijssen MR, Birger Y, Hannah RL, Kinston SJ, Schütte J, Beck D, Knezevic K, Schiby G, Jacob-Hirsch J, et al: Genome-scale expression and transcription factor binding profiles reveal therapeutic targets in transgenic ERG myeloid leukemia. Blood 122: 2694-2703, 2013.

63. Burger MT, Han W, Lan J, Nishiguchi G, Bellamacina C, Lindval M, Atallah G, Ding Y, Mathur M, McBride C, et al: Structure guided optimization, in vitro activity, and in vivo activity of Pan-PIM kinase inhibitors. ACS Med Chem Lett 4: 1193-1197, 2013.

64. Shah N, Pang B, Yeoh KG, Thorn S, Chen CS, Lilly MB and Salto-Tellez M: Potential roles for the PIM1 kinase in human cancer - a molecular and therapeutic appraisal. Eur J Cancer 44: 2144-2151, 2008. 